\title{
Responsabilidad social: voluntariado universitario y comportamiento virtuoso. El caso de una ciudad de Perú
}

\author{
Giusseppe Sarmiento-Peralta ${ }^{1}$, Pedro Severino-González ${ }^{2^{*}}$ y Valentín Santander-Ramírez ${ }^{3}$ \\ (1) Dirección General de Responsabilidad Social, Universidad Nacional Mayor de San Marcos, Lima, Perú \\ (correo-e: giusseppe.sarmiento@unmsm.edu.pe) \\ (2) Facultad de Ciencias Sociales y Económicas, Departamento de Economía y Administración, Universidad Católica del \\ Maule, Talca, Chile (correo-e: pseverino@ucm.cl) \\ (3) Centro de Investigación y Estudios Contables, Facultad Economía y Negocios, Universidad de Talca, Chile \\ (correo-e: valentin.santander@utalca.cl)
}

* Autor a quien debe ser dirigida la correspondencia.

Recibido Ene. 5, 2021; Aceptado Mar. 8, 2021; Versión final Abr. 11, 2021, Publicado Oct. 2021

\begin{abstract}
Resumen
El presente trabajo tiene como propósito explorar la responsabilidad social del estudiante universitario desde la perspectiva del voluntariado como comportamiento virtuoso. Se aplicó un instrumento a una muestra (318 participantes) no probabilística por conveniencia. Se realizó un análisis factorial exploratorio y se examinaron diferencias estadísticamente significativas según características sociodemográficas. Los resultados muestran diferencias significativas según los criterios: años de permanencia en la universidad y según la pertenencia a grupos de voluntariado. Los estudiantes que declaran poseer dos años de permanencia en la universidad expresan una mayor valoración significativa en la dimensión planteamiento del ejercicio profesional desde el compromiso social. Lo anterior, coincide con la mayor valoración expresada por los estudiantes que pertenecen a grupos de voluntariado. En conclusión, las casas de estudios superiores pueden utilizar estrategias asociadas al voluntariado universitario para la formación en responsabilidad social, para lo cual es relevante desarrollar estrategias que procuren la sensibilidad social, comportamiento virtuoso y el compromiso social.
\end{abstract}

Palabras clave: responsabilidad social; estudiante; universidad; comportamiento; voluntariado

\section{Social responsibility: university volunteering and virtuous behaviour. The case of a city in Peru}

\begin{abstract}
The present study aimed to explore the issue of social responsibility of the university student from the perspective of volunteering as virtuous behavior. An instrument was applied to a non-probabilistic sample (318 participants) for convenience. An exploratory factor analysis was performed and statistically significant differences were examined according to socio-demographic characteristics. The results showed significant differences depending on the number of years attending university and on volunteering experience. Students who declared to have two years attending university expressed a significantly higher appreciation for an approach to professional practice with social commitment. This is in agreement with high valuations expressed by students participating in volunteering groups. In conclusion, higher education institutions can use strategies associated with university volunteering to train students on social responsibility by developing strategies that encourage social sensitivity, virtuous behavior, and social commitment.
\end{abstract}




\section{INTRODUCCIÓN}

El voluntariado universitario es una actividad que asume de forma discrecional y potestativa la búsqueda del bien público sin algún tipo de compensación (Paine et al., 2020), lo que contribuye a la rehumanización, al ejercicio de los valores y al desarrollo integral de los estudiantes. Es una herramienta de profunda transformación social y personal. En ese sentido, puede ser considerado como una práctica de la responsabilidad social universitaria (Georgeou y Hass, 2019), producto del reconocimiento del voluntariado como un comportamiento de naturaleza libre, desinteresada y promotora del bien común de la sociedad (Kislyakov et al., 2019). Ahora bien, la responsabilidad social como fundamento de la vida universitaria es ampliamente estudiada desde diversos enfoques que relevan su condición transversal, holística, multidimensional y humanista. Su accionar es un medio transformador de las instituciones de educación superior permitiendo el involucramiento de los actores internos y externos en procesos permanentes de modelamientos organizacionales, asociadas a las funciones misionales de las casas de estudios superiores (Rouhiainen y Vuorisalo, 2019).

Los beneficios del voluntariado universitario favorecen el replanteamiento social de sus educandos debido a las diversas acciones realizadas en alguna comunidad en particular o, bien en algún grupo específico de personas, dotando a los educandos de habilidades personales y sociales (Díaz-lso et al., 2020), gracias al aprendizaje experiencial y vinculadas a actividades curriculares y extracurriculares. Todo lo cual, fortalece la sensibilidad social y la capacidad en generar vínculos como consecuencia del abordaje de preocupaciones sociales (Chiva-Bartoll et al., 2020); conduciendo al desarrollo de competencias que permiten abordar alguna problemática sanitaria, política y medioambiental (Joseph y Carolissen, 2019; Chawłowska et al., 2021). Lo antes señalado ha sido considerado como una estrategia de aprendizaje servicio, política de responsabilidad social universitaria y estrategia educativa (Cabedo et al., 2018).

Por su parte, los centros educativos de nivel superior deben dedicar parte de sus esfuerzos en la formación de profesionales integrales, procurando la generación de propuestas que busquen contribuir a la solución de carencias, en ocasiones, arraigadas en la sociedad (Larrán-Jorge y Andrades-Peña, 2015; González-Gijón et al., 2020). Lo antes indicado, es fortalecido gracias a la incorporación de principios éticos a la vida universitaria; conduciendo al bien común de todo el tejido social (Castillo-Mori et al., 2018; Pianezzi et al., 2020). Dicho involucramiento coadyuva a la sensibilización de los futuros profesionales en correspondencia a los problemas presentes en la sociedad (Kee et al., 2018). En este contexto, según Vallaeys y ÁlvarezRodríguez (2019) la responsabilidad social universitaria incentiva el reconocimiento de las corresponsabilidades de todos los actores que integran una comunidad universitaria, estimulando a la disposición de soportes para la promoción de estrategias pedagógicas; encontrando ejemplos prácticos para su implementación (Ali et al., 2021). Entre dichas estrategias, se encuentran los procesos reflexivos, los que permiten formar un comportamiento socialmente responsable, cuyas manifestaciones buscan la erradicación de la corrupción (Burean, 2019) y la propuesta de reformas profundas basadas en la equidad y transparencia; las que pueden ser emancipadoras de precariedades sociales.

Por otro lado, las manifestaciones sociales en algunas circunstancias pueden ser consideradas como acciones voluntarias. Dichas expresiones asociadas a la responsabilidad social universitaria son investigadas desde un enfoque prosocial, sistémico y ético, como consecuencia de prácticas virtuosas que generan aportes sustanciales y transcendentales a la sociedad (Salcedo-Muñoz et al., 2017), propiciando espacios para la reflexión de los estudiantes sobre sus procederes, métodos y prácticas; conduciendo al aprendizaje profundo, permanente y significativo (Meyer et al., 2019). Todo cual, motiva el comportamiento de prácticas virtuosas, las cuales son desinteresadas, altruistas, equitativas, empáticas y valiosos, las que contribuyen al crecimiento y progreso de la sociedad (Santos et al., 2020). Lo que está en correspondencia con la responsabilidad social universitaria, debido al despliegue de actividades que tributan al progreso económico, político y social (Gallardo-Vázquez et al., 2020).

Es preciso indicar que la vida universitaria ofrece a los estudiantes un conjunto de experiencias que configuran el sentido del yo, la proximidad, las acciones prosociales y la madurez cognitiva (Martí et al., 2011), lo que, asociado a las características propias del voluntariado, conducen al desarrollo del comportamiento denominado socialmente responsable (Cheng y Sikkink, 2020); como consecuencia de un alto grado de consciencia social (Cady et al., 2018) y solidaridad (Müller et al., 2020). Es por ello que, la pregunta de investigación de esta indagación es ¿Cuáles son las responsabilidades sociales de los estudiantes universitarios desde la perspectiva del voluntariado como comportamiento virtuoso en relación a sus características sociodemográficas? Para lo cual, se define el siguiente objetivo de investigación: explorar la responsabilidad social del estudiante universitario desde la perspectiva del voluntariado universitario como comportamiento virtuoso. 


\section{METODOLOGÍA}

Esta sección considera cada una de las decisiones metodológicas propuestas por esta indagación. Se propone el diseño de investigación, los criterios de inclusión de los participantes, las características del instrumento y, finalmente, la estrategia de análisis.

\section{Diseño}

El diseño de la investigación es exploratorio y microsociológica (Hernández et al., 2010), debido a que busca explorar las responsabilidades sociales de los estudiantes universitarios de Perú. Es, además, una indagación cuantitativa y transeccional, debido a que se utiliza para el levantamiento de información, la autoaplicación de un cuestionario con respuesta cerrada.

\section{Participantes}

La muestra es no probabilística por conveniencia, en total se dispone de 318 estudiantes universitarios de una ciudad ubicada en la zona centro de Perú. Para el levantamiento de información se utilizó un instrumento de autosuministración por medio de Google Forms ${ }^{\circledR}$ y difundido a través de diversas redes sociales, producto del Covid-19 que ha provocado una crisis sanitaria de clase mundial. La ventana de observación es entre el 2 de septiembre al 15 de octubre del 2020.

En relación a las características de los sujetos de participación. En cuanto al género corresponde al $50 \%$ para ambas opciones. En relación al territorio, la mayor concentración se encuentra en urbano con $62.6 \%$. En relación al nivel socioeconómico, la mayor cantidad declara recibir un ingreso considerado medio bajo sumando un $41.6 \%$. En correspondencia con los años de permanencia en la universidad, la mayoría declara poseer tres años (24.5\%), seguido de cerca por los que declaran dos años $(22.6 \%)$ y cuatro años $(22.0 \%)$. Ahora bien, en cuanto a la ocupación el 50\% declara solo estudiar y el otro 50\% declara estudiar y trabajar. Finalmente, en relación a la pertenencia a un grupo de voluntariado, la mayor concentración responde de manera afirmativo alcanzando el $65.4 \%$ (ver tabla 1 ).

Tabla 1: Características de los participantes

\begin{tabular}{|c|c|c|c|}
\hline Variable & Criterios & Cantidad & Valores \\
\hline \multirow[t]{2}{*}{ Género } & Hombre & 159 & $50 \%$ \\
\hline & Mujer & 159 & $50 \%$ \\
\hline \multirow[t]{2}{*}{ Territorio } & Urbano & 199 & $62.6 \%$ \\
\hline & Rural & 119 & $37.4 \%$ \\
\hline \multirow[t]{5}{*}{ Nivel socioeconómico familiar (percepción) } & Bajo & 86 & $27 \%$ \\
\hline & Medio bajo & 131 & $41.2 \%$ \\
\hline & Medio & 93 & $29.2 \%$ \\
\hline & Medio alto & 7 & $2.2 \%$ \\
\hline & Alto & 1 & $0.3 \%$ \\
\hline \multirow[t]{5}{*}{ Permanencia (años en la universidad) } & 2 años & 72 & $22.6 \%$ \\
\hline & 3 años & 78 & $24.5 \%$ \\
\hline & 4 años & 70 & $22.0 \%$ \\
\hline & 5 años & 50 & $15.7 \%$ \\
\hline & 6 años & 48 & $15.2 \%$ \\
\hline \multirow[t]{2}{*}{ Ocupación } & Solo estudia & 159 & $50 \%$ \\
\hline & Estudia y trabaja & 159 & $50 \%$ \\
\hline \multirow[t]{2}{*}{ Voluntariado (pertenece a un grupo) } & $\mathrm{Si}$ & 208 & $65.4 \%$ \\
\hline & No & 110 & $34.6 \%$ \\
\hline
\end{tabular}

\section{Instrumento}

El instrumento utilizado en esta indagación fue diseñado por García-Ramos et al. (2016), el que ha sido aplicado en algunos países en Latinoamérica (Severino-González et al., 2020). El instrumento está compuesto por tres secciones: la primera sección considera preguntas filtros que discriminan las características principales que debe poseer el sujeto de investigación, el cual debe ser estudiante de educación superior de alguna universidad ubicada en la ciudad según el alcance geográfico de esta indagación; la segunda sección considera variables sociodemográficas: género, territorio, nivel socioeconómico, años en la universidad, ocupación y voluntariado; la tercera sección, considera el cuestionario, compuesto por 20 variables cuantitativas (ver tabla 1), en cuanto al tipo de repuesta es una escala tipo Likert de 1 a 6 , donde $1=$ valor mínimo de acuerdo y, 6 =valor máximo de acuerdo (ver tabla 2). 
Tabla 2: Instrumento responsabilidad social del estudiante universitario

\begin{tabular}{|c|c|}
\hline Dimensiones & İtems \\
\hline \multirow{5}{*}{$\begin{array}{l}\text { Compromiso } \\
\text { con los demás y } \\
\text { el entorno }\end{array}$} & $\begin{array}{l}\text { V1.Tengo una visión global de la situación actual del mundo y soy consciente de la necesidad } \\
\text { urgente de un desarrollo sostenible. }\end{array}$ \\
\hline & $\begin{array}{l}\text { V2. Esta toma de conciencia aumenta mi interés como universitario en contribuir a la mejora de mi } \\
\text { entorno más cercano. }\end{array}$ \\
\hline & $\begin{array}{l}\text { V3. Me cuestiono cual es mi posicionamiento personal ante las injusticias sociales, ante el dolor } \\
\text { ajeno. }\end{array}$ \\
\hline & $\begin{array}{l}\text { V4. Pongo en práctica mi capacidad de servicio y compromiso con inmigrantes, discapacitados, } \\
\text { indigentes, niños sin recursos, ancianos... }\end{array}$ \\
\hline & $\begin{array}{l}\text { V5. Considero que una de mis obligaciones como persona es ayudar a lo demás, desde el } \\
\text { compromiso social. }\end{array}$ \\
\hline \multirow{5}{*}{$\begin{array}{l}\text { Descubrimiento } \\
\text { personal de los } \\
\text { valores }\end{array}$} & $\begin{array}{l}\text { V6. Creo que el compromiso social se fundamenta en el reconocimiento y respeto de la dignidad } \\
\text { de toda persona. }\end{array}$ \\
\hline & $\begin{array}{l}\text { V7. Reconozco la necesidad de abrirme a los otros, de ponerme en su lugar y buscar el bien } \\
\text { común, por encima de intereses individualistas. }\end{array}$ \\
\hline & $\begin{array}{l}\text { V8. Considero que el cambio personal es un paso previo y necesario para cambiar la realidad que } \\
\text { me rodea. }\end{array}$ \\
\hline & V9. He experimentado en primera persona la felicidad que conlleva el servi \\
\hline & $\begin{array}{l}\text { V10. Considero que la experiencia de darse a los demás es provechosa para descubrir valores } \\
\text { personales. }\end{array}$ \\
\hline \multirow{5}{*}{$\begin{array}{l}\text { Formación de la } \\
\text { responsabilidad } \\
\text { social }\end{array}$} & $\begin{array}{l}\text { V11. Considero que el hecho de ser universitario ayuda a tomar conciencia de la importancia de la } \\
\text { responsabilidad social. }\end{array}$ \\
\hline & $\begin{array}{l}\text { V12. He reflexionado sobre la importancia de no permanecer indiferente o ajeno ante lo que le } \\
\text { sucede a los demás. }\end{array}$ \\
\hline & $\begin{array}{l}\text { V13. Creo que en la medida en que estudie y me prepare a fondo en la universidad, podre a } \\
\text { portar más al cambio social. }\end{array}$ \\
\hline & $\begin{array}{l}\text { V14. La formación que recibiré en la universidad contribuirá en la práctica a que aumente mi } \\
\text { grado de responsabilidad social }\end{array}$ \\
\hline & $\begin{array}{l}\text { V15. Considero que la responsabilidad social es una competencia que se debe trabajar en la } \\
\text { universidad. }\end{array}$ \\
\hline \multirow{5}{*}{$\begin{array}{l}\text { Planteamiento } \\
\text { del ejercicio } \\
\text { profesional } \\
\text { desde el } \\
\text { compromiso } \\
\text { social }\end{array}$} & $\begin{array}{l}\text { V16. Me planteo el ejercicio de mi profesión futura como una profesión de ejercicio orientación al } \\
\text { bien común. }\end{array}$ \\
\hline & $\begin{array}{l}\text { V17. Considero que el buen hacer profesional implica compromiso, trabajo en equipo, constancia, } \\
\text { empatía, tolerancia, honradez y respeto. }\end{array}$ \\
\hline & $\begin{array}{l}\text { V18. Creo que mi realización personal y mi felicidad pasa por ser un profesional comprometido en } \\
\text { la mejora del conjunto de la sociedad }\end{array}$ \\
\hline & $\begin{array}{l}\text { V19. Considero que la acción de un buen profesional tiene repercusión en su entorno más } \\
\text { inmediato y en otros de transcendencia mayor. }\end{array}$ \\
\hline & $\begin{array}{l}\text { V20. Creo que es realista afirmar que desde el ejercicio profesional es posible el compromiso } \\
\text { social. }\end{array}$ \\
\hline
\end{tabular}

\section{Estrategias de análisis}

Una vez levantados los datos por medio de Google Forms® se exportaron al programa SPSS18. Por un lado, se desarrollaron pruebas para la comprobación de la adecuada utilización del análisis factorial exploratorio (AFE). Posteriormente se examinaron las medias, medianas, desviaciones estándar, para luego determinar los coeficientes de consistencia interna y, las correspondientes correlaciones. En cuanto al AFE, se utilizó el método de extracción de componentes principales a través de la rotación Varimax (Hair et al., 2019). Por otro lado, en cuanto al análisis inferencial, se aplicaron pruebas de normalidad para poder determinar los indicadores más adecuados según la naturaleza de la distribución de los datos.

\section{RESULTADOS}

Esta sección presenta los hallazgos relacionados con el análisis factorial exploratorio. Luego se exhiben los resultados de las aplicaciones de los estadísticos descriptivos y, finalmente, se presentan las inferencias de medias.

\section{Análisis factorial exploratorio}

Para la verificación de la adecuada aplicación del análisis factorial exploratorio se desarrollaron las siguientes pruebas, cuyos resultados son los que se presentan a continuación. En cuanto al Kaiser-Meyer-Olkin se obtuvo 0.927 y, en relación a la prueba de esfericidad de Bartlett, se obtuvieron los siguientes valores chi2= 3228.197; $\mathrm{gl}=153$ y p-valor<0.000. Todo lo anterior, según Hair et al. (2019), permite afirmar que es adecuada la aplicación del AFE. 
El cuestionario se redujo a 18 variables, siendo eliminadas las variables V10 y V15, debido a que no cumplen con principios estadísticos y epistemológicos; lo antes indicado está en correspondencia con valores que no son considerados aceptables en comunalidades y, además, producto a la limitada contribución a la claridad en la solución factorial (Hair et al., 2019). La dimensión Compromiso con los demás y el entorno, está compuesta por las variables V1, V2, V3, V4 y V5, la dimensión Formación de la responsabilidad social, está compuesta por las viables V11, V12, V13 y V14, la dimensión Planteamiento del ejercicio profesional desde el compromiso social, está compuesta por V16, V17, V18, V19 y V20 y, finalmente la dimensión Descubrimiento personal de los valores, está compuesta por V6, V7, V8 y V9. Ahora bien, la solución factorial que permite revelar la capacidad que poseen los factores en explicar la variabilidad total de los datos es de un $66,97 \%$, lo que responde a la suma de los porcentajes de la varianza explicada por cada uno de los factores extraídos (Hair et al., 2019), todos los cuales constituyen las dimensiones que integran la encuesta de responsabilidad social del estudiante universitario (ver tabla 3).

Tabla 3: Matriz de componentes rotados de la encuesta de responsabilidad social del estudiante universitario

\begin{tabular}{|c|c|c|c|c|}
\hline \multirow[b]{2}{*}{ Variables } & \multicolumn{4}{|c|}{ Dimensiones } \\
\hline & $\begin{array}{c}\text { Compromiso con } \\
\text { los demás y el } \\
\text { entorno }\end{array}$ & $\begin{array}{l}\text { Formación de la } \\
\text { responsabilidad } \\
\text { social }\end{array}$ & $\begin{array}{l}\text { Planteamiento del ejercicio } \\
\text { profesional desde el } \\
\text { compromiso social }\end{array}$ & $\begin{array}{c}\text { Descubrimiento } \\
\text { personal de los } \\
\text { valores }\end{array}$ \\
\hline V1 & 0.784 & & & \\
\hline V2 & 0.762 & & & \\
\hline V4 & 0.755 & & & \\
\hline V3 & 0.667 & & & \\
\hline V5 & 0.556 & & & \\
\hline V12 & & 0.737 & & \\
\hline V11 & & 0.727 & & \\
\hline V13 & & 0.707 & & \\
\hline V14 & & 0.700 & & \\
\hline V19 & & & 0.782 & \\
\hline V20 & & & 0.769 & \\
\hline V18 & & & 0.659 & \\
\hline V16 & & & 0.579 & \\
\hline V17 & & & 0.546 & \\
\hline V8 & & & & 0.730 \\
\hline V9 & & & & 0.725 \\
\hline V7 & & & & 0.684 \\
\hline V6 & & & & 0.522 \\
\hline
\end{tabular}

\section{Estadísticas descriptivas}

En la tabla 4, se puede observar que en cada uno de los casos los coeficientes de consistencia interna alfa de Cronbach son elevados. Además, se logra identificar que las correlaciones son positivas y, entre algunas dimensiones son moderadas y elevadas. Ahora bien, en cuanto a las valoraciones relacionadas con la media y mediana, los mayores valores se encuentran en la dimensión Planteamiento del ejercicio profesional desde el compromiso social (media=4.27; mediana=4.0), debido a la implicancia que tiene el ejercicio profesional en relación con el tiempo y el entorno, colocando siempre a las personas en el centro de toda gestión. Esta dimensión posee una elevada asociación con la dimensión Compromiso con los demás y el entorno, lo que puede ser producto del rol que poseen los jóvenes en cuanto al liderazgo de procesos transformacionales en virtud del servicio al bien común desde su propio trabajo.

Tabla 4: Media, mediana, desviación estándar y correlaciones entre las dimensiones ( ${ }^{\star *}$. La correlación es significativa al nivel 0.01 , bilateral).

\begin{tabular}{|c|c|c|c|c|c|c|c|c|}
\hline Dimensiones & Media & Mediana & $\begin{array}{l}\text { Des. } \\
\text { estándar }\end{array}$ & Alfa & 1 & 2 & 3 & 4 \\
\hline $\begin{array}{l}\text { 1. Compromiso con los demás y el } \\
\text { entorno }\end{array}$ & 4.10 & 4.0 & 0.930 & 0.858 & 1 & & & \\
\hline $\begin{array}{l}\text { 2. Formación de la responsabilidad } \\
\text { social }\end{array}$ & 4.13 & 4.0 & 0.866 & 0.837 & $0.471^{* *}$ & 1 & & \\
\hline $\begin{array}{l}\text { 3. Planteamiento del ejercicio } \\
\text { profesional desde el compromiso } \\
\text { social }\end{array}$ & 4.27 & 4.0 & 0.827 & 0.872 & $0.618^{* *}$ & $0.483^{* *}$ & 1 & \\
\hline $\begin{array}{l}\text { 4. Descubrimiento personal de los } \\
\text { valores }\end{array}$ & 4.14 & 4.0 & 0.773 & 0.767 & $0.440^{* *}$ & $0.447^{* *}$ & $0.433^{* *}$ & 1 \\
\hline
\end{tabular}




\section{Inferencias de medias}

Para la adecuada examinación de diferencias estadísticamente significativas, se aplicaron pruebas de normalidad considerando cada uno de los criterios que se utilizaron para la caracterización de los sujetos de investigación, en este aspecto se aplicó la prueba de normalidad Kolmogorov-Smirnov y Shapiro-Wilk, la que permite examinar los supuestos de normalidad y homocedasticidad, arrojando en todos los casos que los datos responden a una distribución no normal. Lo que amerita la aplicación de las siguientes pruebas no paramétricas: $\mathrm{H}$ de Kruskal-Wallis y $\mathrm{U}$ Mann Whitney. Los resultados permiten encontrar diferencias de medias según el criterio: permanencia, el que hace referencia a los años en la universidad y, voluntariado, el que considera la pertenencia o no a grupos de voluntariado.

En la tabla 5, se puede observar, según la prueba no paramétrica Kruskal-Wallis, que solo se encontraron diferencias estadísticamente significativas en las dimensiones Compromiso con los demás y el entorno y, Planteamiento del ejercicio profesional desde el compromiso social. En cuanto a la dimensión Compromiso con los demás y el entorno, los mayores valores se encuentran en el grupo de estudiantes que declaran posee dos años en la universidad (media=4.29; mediana=5; $D E=0.911$ ), cuyo valor presenta una zigzagueante disminución a medida que permanecen más años en la universidad. Lo recién señalado, debería estimular a las casas de estudios superiores al desarrollo de estrategias sistemáticas que promueva la sensibilidad social de sus educandos, colocando en práctica servicios comunitarios, compromiso social, ayuda hacia el prójimo; reconociendo en todo momento el respeto a la dignidad humana como analogía al comportamiento virtuoso.

Ahora bien, en cuanto a la dimensión Planteamiento del ejercicio profesional desde el compromiso social, los mayores valores se encuentran en el grupo de estudiantes que declaran posee dos años en la universidad (media $=4.38$; mediana $=5 ; \mathrm{DE}=0.777$ ), seguidos muy de cerca por el grupo de estudiantes que declaran poseer tres años en la universidad (media=4.36; mediana $=4 ; \mathrm{DE}=0.664$ ). En esta dimensión, al igual que en la situación anterior, los valores presentan una zigzagueante disminución a medida que permanecen más años en la universidad, lo que está en correspondencia con los esfuerzos que buscan el reconocimiento del rol del futuro titulado en cuanto a su ejercicio profesional. Todo lo antes indicado es preocupante, debido a que las instituciones de educación superior deben incorporar de manera progresiva estrategias educativas, que permitan aumentar el compromiso del educando con su entorno y su propio planteamiento frente a la sociedad, en cuanto a su compromiso social como futuro profesional; motivando a la materialización de iniciativas de responsabilidad social universitaria, como: aprendizaje servicio, asesorías y capacitaciones comunitarias, semilleros de investigación, prácticas profesionales sociales y voluntariado universitario.

Tabla 5: Media, mediana, desviación estándar por dimensión según años de permanencia en la universidad.

\begin{tabular}{|c|c|c|c|c|c|}
\hline $\begin{array}{l}\text { Permanencia } \\
\text { (años en la } \\
\text { universidad) }\end{array}$ & Estadístico & $\begin{array}{l}\text { Compromiso } \\
\text { con los demás y } \\
\text { el entorno }\end{array}$ & $\begin{array}{l}\text { Formación de la } \\
\text { responsabilidad } \\
\text { social }\end{array}$ & $\begin{array}{c}\text { Planteamiento del } \\
\text { ejercicio profesional } \\
\text { desde el } \\
\text { compromiso social }\end{array}$ & $\begin{array}{c}\text { Descubrimiento } \\
\text { personal de los } \\
\text { valores }\end{array}$ \\
\hline \multirow{3}{*}{2 años } & Media & 4.29 & 4.18 & 4.38 & 4.11 \\
\hline & Mediana & 5 & 4 & 5 & 4 \\
\hline & Desv. estándar & 0.911 & 0.718 & 0.777 & 0.64 \\
\hline \multirow{3}{*}{3 años } & Media & 4.23 & 4.29 & 4.36 & 4.21 \\
\hline & Mediana & 4 & 4 & 4 & 4 \\
\hline & Desv. estándar & 0.821 & 0.686 & 0.664 & 0.589 \\
\hline \multirow{3}{*}{4 años } & Media & 4.03 & 4.13 & 4.29 & 4.14 \\
\hline & Mediana & 4 & 4 & 4 & 4 \\
\hline & Desv. estándar & 0.884 & 0.867 & 0.819 & 0.728 \\
\hline \multirow{3}{*}{5 años } & Media & 3.64 & 3.84 & 3.92 & 3.88 \\
\hline & Mediana & 4 & 4 & 4 & 4 \\
\hline & Desv. estándar & 1.083 & 1.149 & 0.922 & 1.043 \\
\hline \multirow{3}{*}{6 años } & Media & 4.17 & 4.08 & 4.31 & 4.31 \\
\hline & Mediana & 4 & 4 & 5 & 5 \\
\hline & Desv. estándar & 0.883 & 0.942 & 0.971 & 0.903 \\
\hline \multicolumn{2}{|l|}{ p-valor } & 0.003 & 0.374 & 0.02 & 0.095 \\
\hline
\end{tabular}

En la tabla 6, se puede observar según la prueba no paramétrica U Mann Whitney, diferencias estadísticamente significativas en tres dimensiones, las cuales son: Compromiso con los demás y el entorno (media=4.29; mediana=5; $\mathrm{DE}=0.842$ ), Formación de la responsabilidad social ( $m e d i a=4.31$; mediana=4; $\mathrm{DE}=0.703$ ) y Planteamiento del ejercicio profesional desde el compromiso social (media=4.43; mediana $=5$; $\mathrm{DE}=0.663$ ), quedando fuera exclusivamente la dimensión Descubrimiento personal de los valores. Se puede observar que, en todos los casos, los estudiantes que declaran integrar un grupo de voluntariado, los valores son mayores. Todo lo cual, gracias al reconocimiento del compromiso como mecanismo que contribuye a la calidad de vida de grupos que presentan alguna necesidad. Así mismo, es fruto de la importancia que otorgan 
a las problemáticas sociales, lo cual puede ser estimulado con mayor ímpetu a través de la formación en responsabilidad social, debido a su correspondencia con el bien común que deberían tener todos los futuros profesionales, promotores de acciones que tienen su asidero en valores como: trabajo en equipo, dignidad humana, empatía, respeto, compromiso social; evidenciado un comportamiento virtuoso y socialmente responsable.

Tabla 6: Media, mediana, desviación estándar por dimensión según pertenencia a un grupo de voluntariado

\begin{tabular}{|l|l|c|c|c|c|}
\hline \multirow{2}{*}{ Voluntariado } & Estadístico & $\begin{array}{c}\text { Compromiso } \\
\text { con los demás } \\
\text { y el entorno }\end{array}$ & $\begin{array}{c}\text { Formación de la } \\
\text { responsabilidad } \\
\text { social }\end{array}$ & $\begin{array}{c}\text { Planteamiento del } \\
\text { ejercicio profesional } \\
\text { desde el compromiso } \\
\text { social }\end{array}$ & $\begin{array}{c}\text { Descubrimiento } \\
\text { personal de los } \\
\text { valores }\end{array}$ \\
\hline \multirow{3}{*}{ Si pertenece } & Media & 4.29 & 4.31 & 4.43 & 4.2 \\
\cline { 2 - 6 } & Mediana & 5 & 4 & 5 & 4 \\
\cline { 2 - 6 } & Desv. estándar & 0.842 & 0.703 & 0.663 & 0.604 \\
\hline \multirow{2}{*}{$\begin{array}{l}\text { No } \\
\text { pertenece }\end{array}$} & Media & 3.74 & 3.79 & 3.97 & 4.01 \\
\cline { 2 - 6 } & Mediana & 4 & 4 & 4 & 4 \\
\hline pesv. estándar & 0.983 & 1.032 & 1.009 & 0.009 \\
\hline p-valor & 0.000 & 0.000 & 0.000 & 0.46 \\
\hline
\end{tabular}

\section{DISCUSIÓN}

Las dimensiones evaluadas en esta investigación, son similares a los constructos encontrados por SeverinoGonzález et al. (2020). Los resultados indican que los estudiantes universitarios expresan una mayor responsabilidad social en relación a la dimensión Planteamiento del ejercicio profesional desde la perspectiva del compromiso social, lo que está en correspondencia con el voluntariado universitario (Díaz-Iso et al., 2020). Esto se relaciona con los resultados obtenidos por Barajas et al. (2020), particularmente con los hallazgos que dan cuenta del reconocimiento de los compromisos que deben tener los futuros profesionales con la sociedad, en búsqueda del bien común del territorio y la comunidad en general. Lo antes señalado, puede ser impulsado a través del involucramiento del educando en situaciones de contexto en donde se coloque en tensión la necesidad de algún grupo en particular de la sociedad, motivando a la ejecución de acciones que tienen su sustento en valores que evidencian conductas virtuosas.

Por otro lado, considerando los años de permanencia en la universidad, en esta indagación los estudiantes universitarios evidencian una mayor valoración de las prácticas de responsabilidad social en su segundo año; la que disminuye a medida que avanzan en su formación profesional superior. Lo recién señalado, puede ser producto de la débil incorporación de la responsabilidad social en los planes de estudios y, en las estructuras académicas y estratégicas de las casas de estudios superiores (Castillo-Mori et al., 2018). En este contexto, la responsabilidad social universitaria y el voluntariado, podría ser la vía para la ejecución de actividades que aporten al progreso económico, político y social (Gallardo-Vázquez et al., 2020), lo que forja un comportamiento virtuoso y socialmente responsable.

Ahora bien, en relación a la participación en grupos de voluntariado, los estudiantes que participan en agrupaciones de voluntariado demuestran una mayor responsabilidad social con respecto de quienes no realizan actividades de similares propósitos. Lo antes indicado, estimula en los futuros titulados un comportamiento ético y prosocial (Salcedo-Muñoz et al., 2017), lo que fortalecerán los valores que sustentarán el ejercicio profesional (Larrán-Jorge y Andrades-Peña, 2015; González-Gijón et al., 2020). Lo que tributa a la responsabilidad social universitaria, gracias al involucramiento con la comunidad a través de actividades de colaboración virtuosas (Kislyakov et al., 2019). Finalmente, según Santos et al. (2020), algunos estudiantes están dispuestos a contribuir a la sociedad a través de la entrega de soluciones a problemas sociales, como así también, a la defensa de los derechos humanos y la promoción de la participación ciudadana.

\section{CONCLUSIONES}

En relación al trabajo desarrollado y los resultados obtenidos en ésta indagación que busca explorar la responsabilidad social del estudiante universitario de Perú, se pueden presentar las siguientes conclusiones: 1) la formación de los estudiantes universitarios debe responder al desarrollo de competencias integrales, los que iluminen a la sociedad por sus decisiones en donde se identifican capacidades éticas y profesionales, las que podrían mejorar su efectividad según las particularidades sociodemográficas de los educandos; 2) los resultados de esta indagación admiten su utilización para el diseño de modelos, políticas y planes de desarrollo estratégicos educativos, los que pueden contribuir a la disposición de entornos de aprendizajes tanto curriculares como extracurriculares, que coadyuven a la disposición de profesionales socialmente responsables las que deberían considerar las características particulares de los estudiantes según los años de permanencia en la casa de estudios superiores y, la pertenencia o no, a grupos de voluntariado; 3) el voluntariado universitario es una estrategia que se podría insertar en la estructura organizacional de las casas 
de estudios superiores, fortaleciendo el desarrollo de profesionales sensibles a los problemas presentes en la sociedad, motivándolos a una praxis genuina y desinteresada; 4) entre las limitaciones de este trabajo, se encuentra el procedimiento en la suministración del instrumento y, el número de participantes, ya que por los principios de aleatoriedad se ven condicionados producto de la crisis sanitaria y, la cantidad de participantes, restringiendo la aplicación de técnicas estadísticas más robustas; 5) el uso de estos hallazgos, deben poseer el soporte suficiente a través de indagaciones con iguales o similares propósitos para asegurar la eficacia en su utilización, lo que está en correspondencia con el contexto en donde se sitúan los sujetos que se desean investigar o intervenir; 6) finalmente, es preciso que futuras indagaciones pueden considerar una mayor cantidad de características sociodemográficas de los estudiantes y, la aplicación de análisis multivariante que permite explicar causas y efectos entre los constructos que configuran la responsabilidad social del estudiantes y el voluntariado universitario.

\section{AGRADECIMIENTOS}

Este trabajo de investigación fue posible gracias a las iniciativas desarrolladas en Perú y Latinoamérica por el Director General de Responsabilidad Social de la Universidad Nacional Mayor de San Marcos (Perú). Se extiende el presente agradecimiento y homenaje póstumo a la memoria de Doctor José Manuel Yampufé Cornetero (1962-2021).

\section{REFERENCIAS}

Ali, M., Mustapha, I., Osman, S., y Hassan, U., University social responsibility: A review of conceptual evolution and its thematic analysis, https://doi.org/10.1016/j.jclepro.2020.124931, Journal of Cleaner Production, 286, 124931 (2021).

Barajas, J., Benítez, M., y Ramírez, R., Responsabilidad Social del Estudiante en una Universidad Pública de México, http://dx.doi.org/10.37960/rvg.v25i91.33207, Revista Venezolana de Gerencia, 25(91), 1140-1158 (2020).

Burean, T., Democrats on the streets. Drivers of student protest participation in Romania, https://doi.org/10.1285/i20356609v12i1p22, Partecipazione e Conflitto, 12(1), 22-42 (2019).

Cabedo, L., Royo, M., Moliner, L., y Guraya, T., University social responsibility towards engineering undergraduates: The effect of methodology on a service-learning experience. https://doi.org/10.3390/su10061823, Sustainability, 10(6), 1823 (2018).

Cady, S., Brodke, M., Kim, J., y Shoup, Z., Volunteer motivation: A field study examining why some do more, while others do less, https://doi.org/10.1002/jcop.21939, Journal of Community Psychology, 46(3), 281-292 (2018).

Castillo-Mori, Y., Mendoza-Mego, B., Plasencia-Dueñas, E., y Díaz-Vélez, C., Grado de responsabilidad social en estudiantes de una universidad pública, Educación Médica Superior, 32(2), 10-27 (2018).

Chawłowska, E., Staszewski, R., y otros siete autores, Student volunteering as a solution for undergraduate health professions education: lessons from the covid-19 pandemic, https://doi.org/10.3389/fpubh.2020.633888, Frontiers Public Health, 8, 633888 (2021).

Cheng, A., y Sikkink, D., A Longitudinal Analysis of Volunteerism Activities for Individuals Educated in Public and Private Schools, https://doi.org/10.1177/0044118X19861979, Youth and Society, 52(7), 1193-1219 (2020).

Chiva-Bartoll, O., Ruiz Montero, P.J., Capella-Peris, C., y Salvador-García, C., Effects of Service Learning on Physical Education Teacher Education Students' Subjective Happiness, Prosocial Behavior, and Professional Learning, https://doi.org/10.3389/fpsyg.2020.00331, Frontiers in psychology, 11: 331 (2020).

Díaz-Iso, A., Eizaguirre, A., y García-Olalla, A., Understanding the Role of Social Interactions in the Development of an Extracurricular University Volunteer Activity in a Developing Country, http://doi.org/10.3390/ijerph17124422, International Journal of Environmental Research and Public Health, 17(12), 4422 (2020).

Gallardo-Vázquez, D., Folgado-Fernández, J.A., Hipólito-Ojalvo, F., y Valdez-Juárez, L.E., Social responsibility attitudes and behaviors' influence on university students' satisfaction. https://doi.org/10.3390/socsci9020008, Social Sciences, 9(2), 8 (2020).

García-Ramos, J., De La Calle-Maldonado, C., Valbuena-Martínez, M., y De Dios Alija, T., Hacia la validación del constructo "responsabilidad social del estudiante universitario" (RSEU), Bordón. Revista de Pedagogía, 68(3), 41-58 (2016).

Georgeou, N., y Haas, B., Power, Exchange and Solidarity: Case Studies in Youth Volunteering for Development, https://doi.org/10.1007/s11266-019-00103-w, Voluntas, 30(6), 1406-1419 (2019).

González-Gijón, G., Martínez-Heredia, N., Amaro-Agudo, A., y Soriano-Díaz, A., Estudio de los valores en el alumnado que cursa el grado de maestro en educación primaria en las universidades públicas de Andalucía, http://dx.doi.org/10.4067/S0718-50062020000200083, Formación universitaria, 13(2), 83-92 (2020).

Hair Jr, J.F., Gabriel, M., Da Silva, D., y Braga-Junior, S., Development and validation of attitudes measurement scales: fundamental and practical aspects, https://doi.org/10.1108/RAUSP-05-2019-0098, RAUSP Management Journal, 54(4), 490-507 (2019). 
Hernández, R., Fernández, C., y Baptista, C. Metodología de la Investigación, 5ª edición, 58-79. McGraw-Hill, México D.F., México (2010).

Joseph, B.M., y Carolissen, R., Citizenship: A Core Motive for South African University Student Volunteers, https://doi.org/10.1177/1746197918792840, Education, Citizenship and Social Justice, 14(3), 225-240 (2019).

Kee, Y., Li, C., Wang, J., y Kailani, M., Motivations for Volunteering and Its Associations Life Satisfaction: A Latent Profile Approach, http://dx.doi.org/10.1177/0033294117741653, Psychological Reports, 121(5), 932-951 (2018).

Kislyakov, P., Shmeleva, E., y Gowin, O., Contemporary volunteering in the formation of prosocial behaviour of a person, http://dx.doi.org/10.17853/1994-5639-2019-6-122-145, Obrozovanie i Nauka, 21(6), 122-145 (2019).

Larrán-Jorge, M., y Andrades-Peña, F., Analysis of social responsability of universities from different theoretical stances, https://doi.org/10.1016/S2007-2872(15)30005-6, Revista Iberoamericana de Educación Superior, 6(15), 91-107 (2015).

Martí, J., Martí-Vilar, M., y Puerta, I., Towards Neuroresponsibility's model: a perspective of social responsibility since human development, International Journal of Psychological Research, 4(1), 24-28 (2011).

Meyer, M., Neumayr, M., y Rameder, P., Students' Community Service: Self-Selection and the Effects of Participation, https://doi.org/10.1177/0899764019848492, Nonprofit and Voluntary Sector Quarterly, 48(6), 1162-1185 (2019).

Müller, C., Scheffer, A., y Closs, L. Volunteer tourism, transformative learning and its impacts on careers: The case of Brazilian volunteers, https://doi.org/10.1002/jtr.2368, International Journal of Tourism Research, 22(6), $726-738$ (2020).

Paine, A., Allum, C., Beswick, D., y Louh, B., Volunteering and the sustainable development goals: An opportunity to move beyond boundaries, https://doi.org/10.1332/204080520X15884252988018, Voluntary Sector Review, 11(2), 245254 (2020).

Pianezzi, D., Norreklit, H., y Cinquini, L., Academia after virtue? An inquirí into the Moral Character(s) of Academics, https://doi.org/10.1007/s10551-019-04185-w, Journal of Business Ethics, 167(3), 571-588 (2020).

Rouhiainen, H., y Vuorisalo, T., Higher Education teachers' conceptions of sustainable development: implications for interdisciplinary pluralistic teaching, https://doi.org/10.1080/13504622.2019.1657069, Environmental Education Research, 25(12), 1713-1730 (2019).

Salcedo-Muñoz, V., Núñez, L.,, y otros tres autores, Enfoque exploratorio sobre acciones de Responsabilidad Social en universidades del Ecuador: Caso UPSE y UTMACH. Espacios, 38(35), 40-55 (2017).

Santos, G., Marques, C., Justino, E. y Mendes, L., Understanding social responsability's influence on service quality and student satisfaction in Higher Education, https://doi.org/10.1016/j.jclepro.2020.120597, Journal of Cleaner Production, 256, 1-10 (2020).

Severino-González, P., Romero-Argueta, J., Villalobos-Antúnez, J.V., y Garrido-Véliz, V., Social Responsibility of Higher Education Students. Motivations for its Development in Times of Covid-19 in Chile and El Salvador, http://doi.org/10.5281/zenodo.4009788, Utopía y Praxis Latinoamericana, 25, 439-452 (2020).

Vallaeys, F., y Álvarez-Rodríguez, J., Hacia una definición latinoamericana de responsabilidad social universitaria. Aproximación a las preferencias conceptuales de los universitarios, https://doi.org/10.5944/educXX1.19442, Educación XX1, 22(1), 93-116 (2019). 
